\title{
Spin-Dependent Transport through Metallic System with Magnetic Impurities
}

\author{
B.J. Spisak, M. Woloszyn and A. Paja \\ Faculty of Physics and Applied Computer Science \\ AGH - University of Science and Technology \\ al. Mickiewicza 30, 30-059 Kraków, Poland
}

\begin{abstract}
The problem of spin-dependent transport of electrons through a metallic nanostructure is considered. The system consists of non-magnetic metal wire with two magnetic impurities and is connected to two ferromagnetic leads. The differential conductance is calculated by using the transfer matrix method. The spin polarization of the conductance is also obtained. It was found that this polarization is dependent on the spin configuration of magnetic impurities. This dependence can be controlled by the applied bias voltage.
\end{abstract}

PACS numbers: 72.10.Fk, 72.25.Ba, 73.63.Nm

\section{Introduction}

The spin-dependent transport phenomena in nanostructures have attracted much attention in the field of modern electronics with respect to the spin-polarized current and tunneling phenomena $[1,2]$. The control and conservation of the spin-polarized current through the nanodevices form fundamental challenge of spintronics.

In this contribution we consider the effect of spin configuration of two magnetic impurities on the spin-polarized electron transport in a metallic nanosystem. A small number of magnetic impurities allows us to analyze the spin-dependent electron transport in the ballistic regime. Additionally, we assume that the linear size of the one-dimensional nanosystem is much smaller than the spin coherence length. We look for the changes in spin polarization of conductance due to the presence of magnetic impurities. We present the calculations of the differential conductance and the spin polarization of conductance in such nanosystem assuming the spin-dependent current for fixed positions of magnetic impurities and all possible but also fixed configurations of their spins. The apparent changes in the polarization were obtained for some system configurations.

\section{Theoretical model and method of calculations}

The device consists of a non-magnetic metallic wire between two ferromagnetic leads made of the same material. The wire is doped with two magnetic impurities. We assume that the direction of electron spin is fixed by the injection process from the left ferromagnetic lead into the metallic region. The interfaces between ferromagnetic leads and the non-magnetic metallic wire form potential barriers which are caused by the band mismatch between the ferromagnetic leads and the non-magnetic metallic wire and their differences in lattice constants [3]. The Hamiltonian of such system has the form

$$
H=-\frac{\hbar^{2}}{2} \frac{\mathrm{d}}{\mathrm{d} x}\left(\frac{1}{m(x)} \frac{\mathrm{d}}{\mathrm{d} x}\right)+U_{0}(x)+U_{\mathrm{imp}}^{\sigma}(x),
$$

where $m(x)$ is the position-dependent effective mass of conduction electron, the magnetic impurity potential $U_{\mathrm{imp}}^{\sigma}(x)$ is given by the formula

$$
U_{\mathrm{imp}}^{\sigma}(x)=U_{k}^{\sigma} \delta\left(x-X_{k}\right)+U_{l}^{\sigma} \delta\left(x-X_{l}\right),
$$

where $X_{k}$ and $X_{l}$ are the positions of magnetic impurities. We assume $U_{k(l)}^{\sigma}=J_{0} S_{z}\left(X_{k(l)}\right) s_{z}(x)$, where $J_{0}$ is the coupling constant, $S_{z}\left(X_{k(l)}\right)$ is the $z$-component of impurity spin, and $s_{z}$ is the $z$-component of conduction electron spin. The ordinary potential $U_{0}(x)$ has the form

$$
U_{0}(x)= \begin{cases}\mu_{\mathrm{L}}, & x \leq X_{i}, \\ U_{\mathrm{L}}, & x \in\left(X_{i}, X_{j}\right], \\ U, & x \in\left(X_{j}, X_{m}\right], \\ U_{\mathrm{R}}, & x \in\left(X_{m}, X_{n}\right], \\ \mu_{\mathrm{R}}, & x>X_{n},\end{cases}
$$

where the distance $X_{n}-X_{i}$ is equal to the length of the nanosystem, $U_{\mathrm{L}}$ and $U_{\mathrm{R}}$ are the barrier heights of the interfaces, and distances $X_{j}-X_{i}$ and $X_{n}-X_{m}$ correspond to the interface thicknesses.

To investigate the tunneling of spin-polarized electrons through the considered nanosystem we extract the spin-dependent transmission coefficient $T^{\sigma}(E)$ from the solution of the time-independent Schrödinger equation, $H \phi(x)=E \phi(x)$, for the Hamiltonian (1) using the transfer matrix methods (cf. [4]). The knowledge of the transmission coefficient allows us to calculate the spindependent conductance. In the linear response theory the 
spin-dependent conductance is given by the formula [5]:

$$
G^{\sigma}=\frac{e^{2}}{h} \int_{0}^{\infty} \mathrm{d} E T^{\sigma}(E)\left[-\frac{\partial f_{\mathrm{FD}}(E, V)}{\partial E}\right],
$$

where $f_{\mathrm{FD}}(E, V)=\left\{1+\exp \left[(E-e V) /\left(k_{\mathrm{B}} T\right)\right]\right\}^{-1}$ is the Fermi-Dirac distribution function, and the external bias $V=\left(\mu_{\mathrm{L}}-\mu_{\mathrm{R}}\right) / e$.

\section{Results and discussion}

We performed numerical calculations of the spin-polarized current using the typical material parameters of $\mathrm{Co} / \mathrm{Cu} / \mathrm{Co}$ system at $4.2 \mathrm{~K}$. We assumed that the ferromagnetic leads were polarized in parallel, the length of wire was $40 \mathrm{~nm}$ and the wire was doped by two $\mathrm{Mn}^{2+}$ ions. The distance between them was $13 \mathrm{~nm}$ and the value of $U_{k(l)}^{\sigma}$ was $2.75 \mathrm{eV}$ [6]. Figure 1 shows the conductance of spin-up and spin-down conduction electrons as a function of the applied voltage at $4.2 \mathrm{~K}$. The peaks in conductance correspond to the resonant states in the nanosystem. When the energy of spin-polarized electrons is close to the energy of resonant state, their tunneling is observed. The energies of the resonant states are dependent on the length of the nanosystem, thicknesses of the interfaces and the spin configurations of magnetic impurities. The different types of curves present the spin-up conductance for the different spin configurations of the magnetic impurities in the nanosystem.

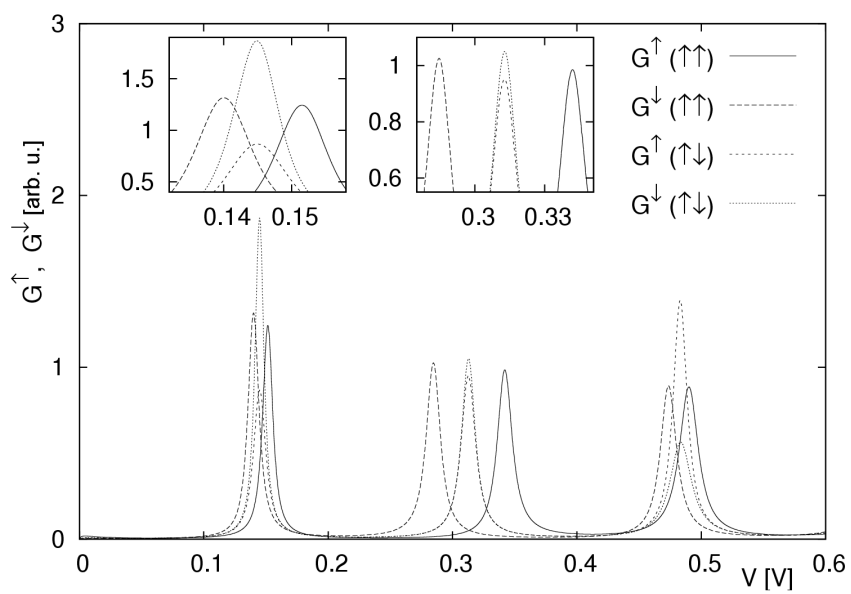

Fig. 1. The spin-up $\left(G^{\uparrow}\right)$ and spin-down $\left(G^{\downarrow}\right)$ conductance for a nanosystem with two magnetic impurities at $T=4.2 \mathrm{~K}$. Double arrows represent the parallel $(\uparrow \uparrow)$ and antiparallel $(\uparrow \downarrow)$ spin configurations of the magnetic impurities. The insets show details of the first two peaks.

The spin polarization of the conductance is defined by the formula [7]:

$$
P=\frac{G_{\mathrm{p}}^{\uparrow}-G_{\mathrm{p}}^{\downarrow}}{G_{\mathrm{p}}^{\uparrow}+G_{\mathrm{p}}^{\downarrow}},
$$

where index p denotes parallel configuration of the magnetization of ferromagnetic leads.
The dependence of the spin polarization of the conductance as a function of applied bias voltage for two different spin configurations of the magnetic impurities is shown in Fig. 2. The dotted line describes the behavior of the conductance when the impurities are antiparallel. The polarization of the conductance defined in Eq. (5) is almost constant except of small deviations in the vicinity of the peaks of conductance shown in Fig. 1. It can be understood because the orientation of electron spins in both currents (spin-up and spin-down) relative to the spin system of impurities is almost identical. The deviations appear in the vicinity of resonances because of small differences in the values of conductances for opposite spins of electrons at the assumed configuration of impurities.

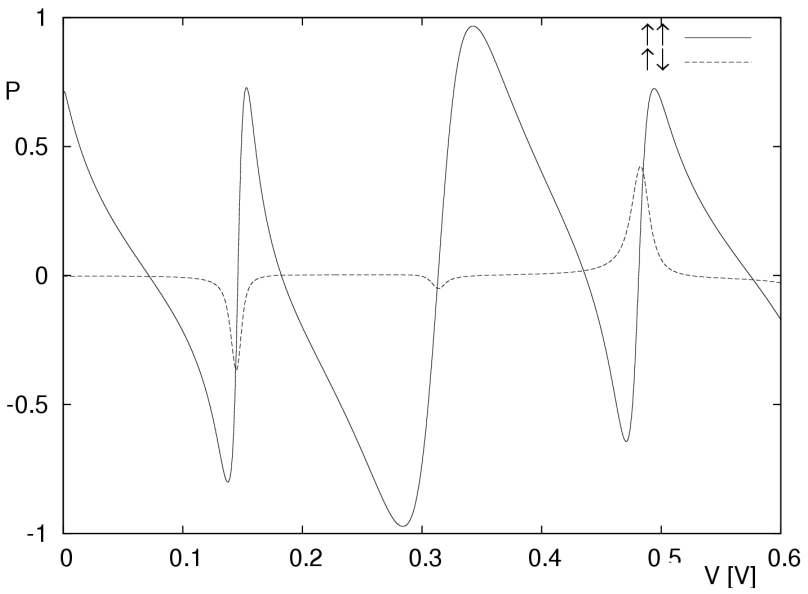

Fig. 2. Dependence of the spin polarization of the conductance on the applied voltage. Arrows represent the relative spin configurations of the magnetic impurities.

The solid line describes the case when spins of both impurities are aligned in parallel. The situation of two spin currents now is quite different: one of them meets spins of impurities parallel to its own, the second one - antiparallel. The energies of interaction apparently differ in these cases and therefore the peaks of respective conductances are shifted (insets in Fig. 1). This gives rise to abrupt changes in the polarization near the positions of resonances, as can be seen in Fig. 2. It means that we could control the effective spin of the current by means of a small change of the bias voltage.

\section{Conclusions}

In this paper we studied the the differential conductance of spin-polarized electrons through the metallic nanowire with two magnetic impurities in the ballistic regime. We have used the transfer matrix method and the Landauer formalism. In the present calculation we neglect the spin-flip processes and the spin correlation effects. The calculated conductance of spin-polarized electrons has pronounced maxima for the values of the applied voltage which correspond to the energies of resonant 
electron states. The spin polarization of the conductance has two different behaviors for two different orientations of magnetic impurities. Both information can be important for design process and fabrication of nanodevices.

\section{Acknowledgments}

Authors would like to thank Mr. M. Ornat for fruitful discussion.

\section{References}

[1] A.M. Bratkovsky, Rep. Prog. Phys. 71, 026502 (2008).
[2] I. Žutić, J. Fabian, S. Das Sarma, Rev. Mod. Phys. 76, 323 (2004).

[3] S.S.P. Parkin, A. Modak, D.J. Smith, Phys. Rev. B 47, 9136 (1993).

[4] P. Harrison, Quantum Wells, Wires and Dots, 1st ed., Wiley, Int. Edition, Chichester 2001, Ch. 2.

[5] S. Datta, Electronic Transport in Mesoscopic Systems, 1st ed., Cambridge University Press, Cambridge 1999, Ch. 2 .

[6] F.W. Smith, Phys. Rev. B 14, 241 (1979).

[7] S. Maekawa, Concepts in Spin Electronics, 1st ed., Oxford University Press, Oxford 2006, Ch. 2. 